\title{
MATRIZES PROGRESSIVAS COLORIDAS DE RAVEN - ESCALA ESPECIAL: NORMAS PARA PORTO ALEGRE, RS
}

\author{
Denise Ruschel Bandeira* \\ Irai Cristina Boccato Alves \\ Angélica Elisa Giacomel" \\ Luciano Lorenzatto ${ }^{\infty}$
}

RESUMO. O Teste das Matrizes Progressivas Coloridas de Raven destina-se à avaliação do desenvolvimento intelectual de crianças de 5 a 11 anos de idade. O presente trabalho teve como objetivo estabelecer normas para as crianças de Porto Alegre - RS. A amostra foi composta por 779 crianças matriculadas em escolas estaduais, de 4 anos e 9 meses a 11 anos e 9 meses. Foi constatado aumento progressivo nas médias de pontos com o aumento da idade e não foram encontradas diferenças entre meninos e meninas. Na comparação com as crianças de escolas públicas de São Paulo, as médias das crianças de Porto Alegre foram mais altas, mas em geral foram inferiores às das escolas particulares de São Paulo. Foram estabelecidas as normas em percentis para cada faixa etária. Os resultados reforçam a necessidade do estabelecimento de normas distintas para as diferentes regiões do Brasil, principalmente em relação aos testes de inteligência..

Palavras-chave: matrizes progressivas coloridas de Raven, avaliação da inteligência, normas.

\section{THE RAVEN'S COLOURED PROGRESSIVE MATRICES: NORMS FOR PORTO ALEGRE, RS}

\begin{abstract}
The Raven's Coloured Progressive Matrices was proposed standards to Porto Alegre (RS) children. The sample was composed by 779 children from Porto Alegre state public schools, aged from 4 years and 9 months to 11 years and 9 months. It was found a progressive increase in average scores as age increased and it was not verified differences between boys and girls. Comparing average scores between children from São Paulo and Porto Alegre public schools we found that the average scores from Porto Alegre children was higher, but they were lower than the scores from São Paulo private schools children. Percentile ranks were obtained to each age level with range of six months. Research results show the need to establish specific norms to different Brazilian regions, mainly concerning intelligence tests.
\end{abstract}

Key words: Raven's Coloured Progressive Matrices, norms, intelligence assessment.

O Teste das Matrizes Progressivas de Raven foi desenvolvido por John C. Raven na Universidade de Dumfries, Escócia, sendo padronizado e publicado em 1938. A forma original, denominada Matrizes Progressivas Standard (Standard Progressive Matrices - SPM), é conhecida no Brasil como Escala Geral. Ela foi "planejada para abranger todas as faixas de desenvolvimento intelectual, desde o momento em que a criança é capaz de compreender a idéia de encontrar o pedaço que falta para completar um desenho" (Angelini, Alves, Custódio, Duarte \& Duarte, 1999, p. XVII).

* Docente do Programa de Pós-Graduação em Psicologia do Desenvolvimento do Instituto de Psicologia da Universidade Federal do Rio Grande do Sul.

\# Docente de Graduação e de Pós-Graduação do Instituto de Psicologia da Universidade de São Paulo e Coordenadora do LITEP - Laboratório Interdepartamental de Técnicas de Exame Psicológico.

II Bolsista PIBIC/CNPq durante a realização da pesquisa, atualmente cursando o Mestrado em Psicologia Social no Instituto de Psicologia da Universidade Federal do Rio Grande do Sul.

x Bolsista PIBIC/CNPq durante a realização da pesquisa e atualmente Mestre em Psicologia do Desenvolvimento pelo Instituto de Psicologia da Universidade Federal do Rio Grande do Sul.

Os autores agradecem o apoio do Centro Editor de Testes Psicológicos, que forneceram todo o material para a pesquisa. 
Em 1947, o autor desenvolveu mais duas escalas, as Matrizes Progressivas Coloridas (Coloured Progressive Matrices - CPM) e as Matrizes Progressivas Avançadas (Advanced Progressive Matrices - APM). A primeira, para ser empregada com crianças pequenas, pessoas idosas e deficientes mentais, destina-se à faixa de 5 a 11 anos, no que se refere a crianças. A segunda, para testar pessoas com capacidade intelectual superior à média, é mais usada para pessoas com escolaridade universitária.

As Matrizes Progressivas Coloridas são conhecidas no Brasil como Escala Especial, que é constituída por três séries de 12 itens: A, Ab e B. Os itens estão dispostos em ordem de dificuldade crescente em cada série, sendo cada série mais difícil do que a série anterior. No início de cada série são sempre colocados itens mais fáceis, cujo objetivo é introduzir o examinando num novo tipo de raciocínio, que vai ser exigido para os itens seguintes. Os itens consistem em um desenho ou matriz com uma parte faltando, abaixo do qual são apresentadas seis alternativas, uma das quais completa a matriz corretamente. $\mathrm{O}$ examinando deve escolher uma das alternativas como a parte que falta.

A escala colorida recebe este nome porque a maior parte de seus itens são impressos com um fundo colorido, cujo objetivo é atrair a atenção e motivar as crianças pequenas. Esta escala foi revista em 1956 por seu autor, que modificou a ordem de dois itens, alterou o desenho de algumas alternativas de itens e mudou as alternativas de alguns itens de posição. Desde essa revisão o teste não foi mais modificado.

$\mathrm{O}$ teste foi elaborado tendo como base $\mathrm{o}$ referencial da teoria bifatorial de Charles Spearman e tem como objetivo avaliar o que o autor define como capacidade intelectual geral - fator " $g$ ". Na verdade, as Matrizes Progressivas pretendem avaliar um dos componentes do fator " $g$ ", a capacidade edutiva. O outro componente é a capacidade reprodutiva, que é avaliada por testes de vocabulário, como as escalas Mill Hill e Crichton, não publicadas no Brasil.

A capacidade edutiva consiste em extrair novos insights (compreensões) e informações do que já é percebido ou conhecido.

As Matrizes medem a capacidade de eduzir relações... porque as variáveis entre as quais as relações devem ser vistas, não são óbvias em si mesmas (Angelini, Alves, Custódio, Duarte, \& Duarte 1999, p. 5).

A relação precisa ser descoberta para que as variáveis possam ser reconhecidas. Portanto a capacidade edutiva relaciona-se à capacidade "de extrair significado de uma situação confusa, de desenvolver novas compreensões, de ir além do que é dado para perceber o que não é imediatamente óbvio, de estabelecer constructos", principalmente não verbais (p. 3).

A capacidade reprodutiva relaciona-se ao domínio, à lembrança e à reprodução de materiais, em geral verbais, que constituem a base cultural de conhecimentos. É avaliada principalmente por testes de vocabulário.

Em função do referencial teórico do qual deriva, o Teste de Raven avalia a inteligência medida pelo seu produto final, ou seja, pelos resultados (Davidoff, 1983). Dessa forma, desconsidera os processos ou diferenças qualitativas que interferem nas respostas dos indivíduos.

As normas para a escala de 1947, obtidas em Dumfries com uma amostra de 608 crianças, foram publicadas pelo seu autor em 1949. Em 1982, houve uma nova padronização, com uma amostra de 598 crianças, usando o teste revisto. No Manual do CPM publicado em 1995, Raven, Court e Raven apresentam diversos estudos normativos recentes, realizados em diversos países. Em 1984, Roberts testou crianças de uma escola no Sudeste da Inglaterra e encontrou resultados muito semelhantes aos da padronização de 1982, feita em Dumfries. Entre os estudos normativos citados, encontram-se o de Reddington e Jackson, em 1988, na Austrália; o de Schmidtke, Schaller e Becker, em 1978, e o de Guthke, em 1986, na Alemanha; o de Ferjencik, em 1985, na Eslováquia; o de Van bom, na Holanda em 1986; o de Gehriger, em 1970, e o de Martinolli, em 1989, na Suíça; o de Kahn, Spears e Rivera, em 1977 em Porto Rico, o de Dolores Munoz numa área isolada das montanhas do Peru em 1993; o de Angelini, Alves, Custódio e Duarte, em São Paulo em 1988; o da Espanha em 1994 e o dos Estados Unidos em 1986.

As normas nacionais americanas foram obtidas em vários locais do país e os dados foram ponderados para refletir a proporção de crianças que viviam nas diversas regiões do país. Essas normas indicaram resultados mais baixos do que os britânicos, mas variaram consideravelmente entre os distritos escolares, sendo que os resultados foram mais altos, conforme aumentava o nível socioeconômico das amostras, com resultados mais baixos para as áreas rurais. Os autores chamam a atenção para a necessidade de normas adequadas para o contexto em que vão ser usadas.

No Brasil foram feitas algumas pesquisas normativas com a forma de 1947 do CPM, destacando-se entre elas a de Angelini, Rosamilha e 
Almeida (1966) e a de Jacquemin e Xavier (1982). O estudo de Angelini, Rosamilha e Almeida foi realizado com 505 crianças entre 7 e 11 anos de idade, na região Centro-Sul do Brasil, das cidades de Belo Horizonte, São Paulo, Rio de Janeiro e Americana. A pesquisa de Jacquemin e Xavier foi realizada com 1131 crianças de 5 a 11 anos, da cidade de Ribeirão Preto, com o nível de escolaridade compatível com a idade, excluindo assim as crianças atrasadas ou adiantadas em relação à escolaridade.

Posteriormente, Jacquemin e Xavier (1984) realizaram com a mesma amostra um estudo da fidedignidade pelo método das metades aplicando a correção de Spearman-Brown. Os coeficientes variaram de 0,45 a 0,93 para as diversas idades, sendo de 0,90 para a amostra total.

Com a forma de 1947, também foi realizada a pesquisa de Pasquali, Wechsler e Bensusan (2002), que examinaram a validade de construto do teste com uma amostra de crianças do Distrito Federal, através da análise fatorial. Constataram a presença de quatro fatores e um fator geral, constituído por 25 dos 36 itens do teste. No entanto, esses resultados não podem ser considerados para a forma atual do teste, pois a forma de 1956 sofreu várias modificações. Foram alteradas as posições de várias alternativas em 11 itens, dois itens tiveram sua ordem trocada (A11 e A12) e um item sofreu modificação no desenho em uma das alternativas (Angelini e cols., 1999). Dessa forma, não é possível saber qual o efeito dessas alterações sobre a estrutura fatorial do teste.

Empregando a forma de 1956, pode ser citada a pesquisa de Bandeira e Hutz (1994), que investigaram o uso conjunto do Desenho da Figura Humana, do Bender e do Raven como preditores do rendimento escolar na primeira série. Os resultados indicaram que, embora o teste de Raven tenha apresentado correlações significantes com o rendimento escolar, não contribuía de forma significativa na predição do rendimento escolar, quando combinado com os outros dois testes.

A revisão da escala colorida também foi usada como parâmetro para estabelecer a validade de critério nas pesquisas de padronização de vários testes. Entre eles podem ser citados a Escala de Maturidade Mental Colúmbia (Alves e Duarte, 1993), o Teste de Inteligência Não Verbal para Crianças - R2 (Rosa e Alves, 2000) e a Escala Wechsler de Inteligência para Crianças - WISC III (Figueiredo, 2002).

O caderno do teste referente à revisão de 1956 foi publicado em 1988 pela Casa do Psicólogo, com um manual abreviado (Raven, Raven \& Court,1988), que incluía as normas da padronização realizada na cidade de São Paulo (SP) por Angelini, Alves, Custódio e Duarte. Esse manual foi substituído pela sua versão atual (Angelini, Alves, Custódio, Duarte e Duarte, 1999), publicada pelo Centro Editor de Testes e Pesquisas em Psicologia (CETEPP), que desde 1992 também é responsável pela publicação dos cadernos de aplicação do teste.

A pesquisa de padronização do teste foi feita na cidade de São Paulo, pela dificuldade de se conduzir uma pesquisa nacional, em função da grande extensão territorial do Brasil, bem como pela grande diversidade cultural existente nas diferentes regiões do país, o que leva à necessidade de construção de normas regionais.

A primeira fase da pesquisa de padronização foi feita com uma amostra de 1417 sujeitos, considerandose as variáveis sexo, idade e tipo de escola. As idades variaram de 4 anos e 9 meses a 11 anos e 9 meses, divididas em 14 faixas etárias, com amplitude de 6 meses, sendo aproximadamente metade de cada sexo. $\mathrm{O}$ tipo de escola foi incluído como uma forma de controle do nível socioeconômico da amostra, procurando-se manter na amostra a mesma proporção de sujeitos que a existente nas matrículas dos três tipos de escola (particular, municipal e estadual), de acordo com as estatísticas oficiais. As normas obtidas foram publicadas no manual de 1988 (Raven, Raven \& Court, 1988).

$\mathrm{Na}$ segunda fase da pesquisa, a amostra original foi complementada com mais 130 sujeitos nas faixas de 11 e 11,5 anos, que cursavam a $5^{\mathrm{a}}$ e $6^{\mathrm{a}}$ séries do primeiro grau, uma vez que a amostra anterior incluía crianças apenas até a $4^{\mathrm{a}}$ série. A amostra ficou composta então de 1547 sujeitos e foi denominada como Amostra Geral.

Tendo-se em vista que a comparação dos resultados das crianças em função do tipo de escola indicou que havia diferenças estatisticamente significantes entre as médias das crianças de escolas particulares em relação às das escolas municipais e estaduais (públicas), decidiu-se fazer tabelas de normas para esses dois tipos de escola. Como a amostra de crianças de escolas particulares era insuficiente para se estabelecerem normas, foi coletada uma amostra complementar nas escolas particulares de 361 crianças, totalizando a amostra final 1908 crianças.

Não foram elaboradas tabelas separadas para meninos e meninas porque foi constatado não haver diferenças estatisticamente significantes nos resultados em função do sexo. As pesquisas relatadas no manual de Raven, Court e Raven de 1995 não fazem 
referência a diferenças nos resultados quanto aos sexos na literatura internacional.

No manual brasileiro de 1999, também foram feitas comparações dos resultados da padronização com as pesquisas brasileiras anteriores e com as de Raven em Dumfries. Nessas comparações foi observado que os resultados da padronização eram superiores aos da pesquisa de 1966 e inferiores aos da pesquisa de Ribeirão Preto, bem como eram inferiores às normas de Dumfries de 1982. Isto mostrou a existência de diferenças entre as diversas normas e a necessidade de fazer pesquisas em outras regiões do país.

As duas tabelas de normas obtidas na Suíça em 1970 e 1989 mostraram um aumento nos escores médios por idade num período de aproximadamente 20 anos, semelhante ao que foi encontrado na comparação das normas de 1947 e 1982 de Dumfries, indicando a necessidade de contínua atualização das normas (Raven, Court \& Raven, 1995). O mesmo foi observado na comparação entre as normas de 1966 e 1988 no Brasil.

Além da necessidade de atualização de normas, Alves (1998) aponta variáveis que influenciam os resultados dos testes de inteligência. No Raven, especialmente, a autora aponta para a influência do nível socioeconômico, que pode variar de região para região. Considerando a necessidade de estabelecer normas regionais para as diversas regiões do Brasil, o presente trabalho tem como objetivo apresentar os resultados relativos às crianças da cidade de Porto Alegre, no Rio Grande do Sul, e compará-las com os resultados da padronização paulistana.

\section{MÉTODO}

\section{Amostra}

Participaram deste estudo 779 crianças, matriculadas em escolas da rede pública estadual desde o Nível A do Ensino Infantil à quinta série do Ensino Fundamental. Dentre estas, 386 são do sexo masculino e 393 do sexo feminino. As crianças foram distribuídas em 14 faixas etárias com amplitude de seis meses, dos 4 anos e 9 meses aos 11 anos e 9 meses. Os limites das faixas etárias foram estabelecidos da mesma forma que na pesquisa realizada em São Paulo, da seguinte forma: para a faixa etária de 5 anos, consideram-se os limites de 4 anos e 9 meses a 5 anos, 2 meses e 29 dias. Para a faixa de 5,5 anos, os limites de 5 anos e 3 meses a 5 anos, 8 meses e 29 dias, e assim sucessivamente. A Tabela 1 apresenta a distribuição de freqüência por faixas etárias e sexo, sendo que se procurou manter um número semelhante de meninos e meninas em cada faixa etária.

Tabela 1. Frequiências de meninos e meninas conforme as faixas etárias.

\begin{tabular}{lccc}
\hline Faixas etárias & Meninos & Meninas & Total \\
\hline 5 & 19 & 31 & 50 \\
5,5 & 25 & 26 & 51 \\
6 & 34 & 32 & 66 \\
6,5 & 28 & 31 & 59 \\
7 & 29 & 26 & 55 \\
7,5 & 22 & 28 & 50 \\
8 & 27 & 28 & 55 \\
8,5 & 25 & 30 & 55 \\
9 & 32 & 33 & 65 \\
9,5 & 32 & 32 & 64 \\
10 & 28 & 24 & 52 \\
10,5 & 30 & 25 & 55 \\
11 & 28 & 22 & 50 \\
11,5 & 27 & 25 & 52 \\
\hline Total & $\mathbf{3 8 3}$ & $\mathbf{3 9 3}$ & $\mathbf{7 7 9}$ \\
\hline
\end{tabular}

\section{Instrumento}

O instrumento utilizado foi o Teste de Matrizes Progressivas Coloridas de Raven ou Escala Especial, publicado em 1988. O material foi constituído do caderno de aplicação, folha de respostas e lápis para anotação.

\section{Procedimento}

Conforme a listagem das escolas cadastradas de Porto Alegre, recebida da Secretaria de Educação e Cultura (SEC), foram sorteadas 27 escolas estaduais, sendo 11 escolas de Ensino Infantil e 16 escolas de Ensino Fundamental. Nesta distribuição, procurou-se respeitar a proporção de escolas segundo as três categorias - estadual, municipal e particular - existentes em Porto Alegre. Dentre os órgãos competentes, somente a SEC, que controla as escolas estaduais, autorizou a realização da pesquisa. A Secretaria de Educação do Município não autorizou a realização nas escolas municipais e as particulares consultadas também não autorizaram.

Em cada escola estadual de Ensino Infantil, foram sorteadas para participar 16 crianças e em cada escola estadual de Ensino Fundamental, 40 crianças, segundo os critérios do projeto paulista (Alves, s/d). Todas foram escolhidas aleatoriamente através de sorteio, 
pelas listas de matrículas fornecidas pela direção de cada escola.

Após o sorteio da amostra e uma vez obtido o consentimento do professor e da criança para a retirada desta da sala de aula, o aplicador conduzia uma turma de, no máximo, 8 crianças, para uma sala especial, disponibilizada pela própria escola, onde a testagem era realizada. As instruções foram dadas coletivamente, num primeiro momento, e individualmente, conforme fosse necessário. Contudo, todas as crianças da Escola Infantil, bem como as que não apresentavam condições de anotar as respostas na folha de respostas, foram testadas individualmente, de acordo com as instruções padronizadas.

\section{RESULTADOS}

Para a análise estatística dos resultados, foi utilizado o programa SPSS. A análise de variância demonstrou não haver diferença significante entre as médias dos dois sexos $[F(777,1)=3,01$ e $p=0,083]$. Os meninos apresentaram média de 21,77 $(D P=5,93)$ e as meninas 21,05 $(D P=5,75)$. Por essa razão, decidiu-se reunir os dados referentes ao dois sexos, sendo realizada uma nova análise de variância para determinar se havia diferenças entre as idades. O resultado constatou um valor para $F(765,13)=61,014$ significante a 0,001, o que indica diferenças significantes estatisticamente em função da idade. Uma análise de seguimento de Tukey mostrou quais faixas etárias se diferenciaram. As médias de acertos para cada faixa etária, assim como os desvios-padrão e os resultados do teste de Tukey são apresentadas na Tabela 2.

Tabela 2. Médias e desvios-padrão de pontos para cada faixa etária.

\begin{tabular}{cccc}
\hline Faixa Etária & $\boldsymbol{X}$ & $\boldsymbol{D P}$ & $\boldsymbol{N}$ \\
\hline 5 & $14,8^{\mathrm{a}}$ & 3,03 & 50 \\
5,5 & $15,4^{\mathrm{a}, \mathrm{b}}$ & 3,06 & 51 \\
6 & $17,2^{\mathrm{a}, \mathrm{b}, \mathrm{c}}$ & 3,92 & 50 \\
6,5 & $17,5^{\mathrm{b}, \mathrm{c}, \mathrm{d}}$ & 3,99 & 59 \\
7 & $18,9^{\mathrm{c}, \mathrm{d}}$ & 3,82 & 55 \\
7,5 & $19,3^{\mathrm{c}, \mathrm{d}}$ & 4,21 & 50 \\
8 & $20,1^{\mathrm{d}, \mathrm{e}}$ & 4,76 & 55 \\
8,5 & $22,1^{\mathrm{e}, \mathrm{f}}$ & 5,22 & 55 \\
9 & $24,3^{\mathrm{f}, \mathrm{g}}$ & 4,25 & 65 \\
9,5 & $24,6^{\mathrm{f}, \mathrm{g}}$ & 3,94 & 64 \\
10 & $25,0^{\mathrm{g}}$ & 4,04 & 52 \\
10,5 & $25,3^{\mathrm{g}}$ & 4,27 & 55 \\
11 & $26,6^{\mathrm{g}, \mathrm{h}}$ & 4,70 & 50 \\
11,5 & $28,6^{\mathrm{h}}$ & 4,04 & 52 \\
\hline Total & $\mathbf{2 1 , 4 1}$ & $\mathbf{5 , 8 5}$ & $\mathbf{7 7 9}$ \\
\hline
\end{tabular}

$\mathrm{a}, \mathrm{b}, \mathrm{c}, \mathrm{d}, \mathrm{e}, \mathrm{f}, \mathrm{g}$ e h: médias com letras iguais não se diferenciaram estatisticamente conforme teste de Tukey, a $\mathrm{p}<0,05$
Como o teste de Tukey não mostrou diferença significativa entre todas as faixas etárias, indicando não haver necessidade de médias a cada seis meses, optou-se por reduzir o número de faixas de quatorze para sete, com amplitude de um ano. Dessa forma, a faixa etária de 5 anos compreendeu os limites de 4 anos e 9 meses a 5 anos, 8 meses e 29 dias, a de 6, os limites de 5 anos e 9 meses a 6 anos, 8 meses e 29 dias. Com essa nova distribuição das faixas etárias, uma análise de variância foi realizada indicando a diferença significativa entre as mesmas $[F(772,6)=128,87$ a $p<0,0001]$. O teste de Tukey mostrou haver diferença significativa entre todas as faixas etárias, menos entre as de 9 e 10 anos. A partir desses resultados, optou-se por estabelecer as normas para Porto Alegre de forma diferente do proposto por Angelini e colaboradores (1999). A Tabela 3 apresenta esses resultados.

Tabela 3. Normas em percentis, médias e desvios padrão para as crianças de Porto Alegre por faixa etária.

\begin{tabular}{cccccccc}
\hline & \multicolumn{7}{c}{ Faixa Etária } \\
\cline { 2 - 8 } Percentil & $\mathbf{4 ; 9} \mathbf{a}$ & $\mathbf{5 ; 9} \mathbf{a}$ & $\mathbf{6 ; 9} \mathbf{a}$ & $\mathbf{7 ; 8} \mathbf{a}$ & $\mathbf{8 ; 9} \mathbf{a}$ & $\mathbf{9 ; 9} \mathbf{a}$ & $\mathbf{1 0 ; 9} \mathbf{a}$ \\
& $\mathbf{5 ; 8}$ & $\mathbf{6 ; 8}$ & $\mathbf{7 ; 8}$ & $\mathbf{8 ; 8}$ & $\mathbf{9 ; 8}$ & $\mathbf{1 0 ; 8}$ & $\mathbf{1 1 ; 8}$ \\
\hline 1 & 10 & 11 & 11 & 13 & 14 & 16 & 17 \\
5 & 11 & 12 & 13 & 13 & 18 & 18 & 20 \\
10 & 11 & 12 & 14 & 14 & 19 & 20 & 21 \\
20 & 12 & 13 & 15 & 16 & 20 & 21 & 24 \\
25 & 13 & 14 & 16 & 16 & 21 & 21 & 25 \\
30 & 13 & 14 & 17 & 17 & 22 & 22 & 25 \\
40 & 14 & 16 & 18 & 19 & 24 & 24 & 26 \\
50 & 15 & 17 & 19 & 21 & 25 & 26 & 28 \\
60 & 15 & 18 & 20 & 23 & 26 & 27 & 29 \\
70 & 16 & 19 & 21 & 24 & 27 & 28 & 30 \\
75 & 17 & 20 & 22 & 25 & 28 & 28 & 32 \\
80 & 18 & 21 & 23 & 26 & 28 & 29 & 32 \\
90 & 20 & 23 & 25 & 28 & 30 & 30 & 34 \\
95 & 21 & 25 & 25 & 29 & 30 & 32 & 35 \\
99 & 23 & 25 & 28 & 31 & 32 & 34 & 35 \\
\hline Média & $\mathbf{1 5 , 0 9}$ & $\mathbf{1 7 , 3 2}$ & $\mathbf{1 9 , 0 9}$ & $\mathbf{2 1 , 0 9}$ & $\mathbf{2 4 , 4 5}$ & $\mathbf{2 5 , 1 6}$ & $\mathbf{2 7}, \mathbf{6 1}$ \\
DP & $\mathbf{3 , 0 4}$ & $\mathbf{3 , 9 4}$ & $\mathbf{4 , 0 0}$ & $\mathbf{5 , 0 7}$ & $\mathbf{4 , 0 9}$ & $\mathbf{4 , 1 5}$ & $\mathbf{4 , 4 7}$ \\
\hline
\end{tabular}

Para fins de comparação entre as médias obtidas em Porto Alegre e as médias obtidas pelos vários grupos de São Paulo, é apresentada a Tabela 4. Estes resultados podem ser comparados com os da Tabela 2. Ainda, na Figura 1, podem ser observadas as curvas polidas obtidas a partir das médias por idade da amostra do Rio Grande do Sul comparada com as das escolas públicas e as de particulares de São Paulo. Para que o nível de significância das diferenças pudesse ser verificado, foram realizados testes " $t$ " da comparação das médias dos diversos subgrupos de São Paulo com a amostra de Porto Alegre. 
Tabela 4. Médias e desvios-padrão dos diversos grupos de São Paulo.

\begin{tabular}{|c|c|c|c|c|c|c|c|c|c|c|c|c|}
\hline \multirow{2}{*}{$\begin{array}{c}\text { Faixa } \\
\text { Etária }\end{array}$} & \multicolumn{3}{|c|}{ Estadual -SP } & \multicolumn{3}{|c|}{ Municipal - SP } & \multicolumn{3}{|c|}{ Pública - SP } & \multicolumn{3}{|c|}{ Particular - SP } \\
\hline & $\mathbf{X}$ & DP & $\mathbf{N}$ & $\mathbf{x}$ & DP & $\mathbf{N}$ & $\mathbf{X}$ & DP & $\mathbf{N}$ & & & \\
\hline 5 & & & & 11,8 & 2,6 & 42 & 11,8 & 2,6 & 42 & 14,9 & 3,2 & 32 \\
\hline 5,5 & & & & 13,7 & 3,6 & 60 & 13,7 & 3,6 & 60 & 15,5 & 3,0 & 36 \\
\hline 6 & 15,5 & 2,1 & 2 & 13,6 & 2,3 & 56 & 13,7 & 2,3 & 58 & 16,9 & 3,5 & 41 \\
\hline 6,5 & 13,9 & 2,7 & 12 & 15,0 & 3,3 & 60 & 14,8 & 3,2 & 72 & 18,7 & 4,5 & 45 \\
\hline 7 & 16,6 & 4,8 & 55 & 15,5 & 3,7 & 38 & 16,2 & 4,5 & 93 & 20,9 & 3,8 & 38 \\
\hline 7,5 & 16,7 & 5,1 & 59 & 17,4 & 5,9 & 25 & 16,2 & 5,3 & 84 & 21,3 & 5,2 & 47 \\
\hline 8 & 17,8 & 5,9 & 52 & 20,6 & 6,2 & 19 & 18,6 & 6,1 & 71 & 23,7 & 5,9 & 49 \\
\hline 8,5 & 18,5 & 5,8 & 66 & 18,9 & 4,9 & 28 & 18,6 & 5,5 & 94 & 24,9 & 5,4 & 56 \\
\hline 9 & 19,9 & 6,3 & 60 & 20,4 & 6,6 & 28 & 20,0 & 6,4 & 88 & 25,9 & 4,9 & 56 \\
\hline 9,5 & 19,2 & 6,8 & 69 & 20,3 & 5,8 & 23 & 19,5 & 6,5 & 92 & 26,6 & 5,3 & 62 \\
\hline 10 & 22,0 & 6,9 & 70 & 20,0 & 5,8 & 23 & 21,5 & 6,6 & 93 & 28,4 & 4,7 & 62 \\
\hline 10,5 & 22,1 & 6,2 & 52 & 23,5 & 6,4 & 26 & 22,6 & 6,2 & 78 & 29,5 & 5,2 & 64 \\
\hline 11 & 25,6 & 6,5 & 106 & 24,4 & 6,3 & 33 & 25,3 & 6,4 & 139 & 31,6 & 3,2 & 66 \\
\hline 11,5 & 26,0 & 7,2 & 112 & 23,0 & 7,1 & 26 & 25,4 & 7,3 & 138 & 29,8 & 6,0 & 52 \\
\hline Total & & & 715 & & & 487 & & & 1202 & & & 706 \\
\hline
\end{tabular}



Figura 1. Comparação entre as médias de pontos por idade de Porto Alegre e São Paulo.

Tabela 5. Resultados dos testes t dos diversos grupos de São Paulo com os do Rio Grande do Sul.

\begin{tabular}{lcccc}
\hline \multirow{2}{*}{$\begin{array}{l}\text { Faixa } \\
\text { Etária }\end{array}$} & $\begin{array}{c}\text { Estadual - RS } \\
\mathbf{x}\end{array}$ & $\begin{array}{c}\text { Estadual - RS } \\
\mathbf{x}\end{array}$ & $\begin{array}{c}\text { Estadual - RS } \\
\mathbf{x}\end{array}$ & $\begin{array}{c}\text { Estadual - RS } \\
\mathbf{x}\end{array}$ \\
\hline 5 & - & $5,055^{*}$ & $5,055^{*}$ & 0,139 \\
5,5 & - & $2,665^{*}$ & $2,665^{*}$ & 0,150 \\
6 & - & $5,624^{*}$ & $5,490^{*}$ & 0,381 \\
6,5 & $3,719^{*}$ & $3,690^{*}$ & $4,173^{*}$ & 1,400 \\
7 & $2,755^{*}$ & $4,249^{*}$ & $3,856^{*}$ & $2,461^{*}$ \\
7,5 & $2,889^{*}$ & 1,411 & $3,705^{*}$ & $2,052^{*}$ \\
8 & $2,191^{*}$ & 0,313 & 1,538 & $3,365^{*}$ \\
8,5 & $3,561^{*}$ & $2,710^{*}$ & $3,842^{*}$ & $2,753^{*}$ \\
9 & $4,503^{*}$ & $2,833^{*}$ & $4,955^{*}$ & 1,887 \\
9,5 & $5,610^{*}$ & $3,227^{*}$ & $6,050^{*}$ & $2,379^{*}$ \\
10 & $2,985^{*}$ & $3,677^{*}$ & $3,929^{*}$ & $4,117^{*}$ \\
10,5 & $3,063^{*}$ & 1,280 & $2,951^{*}$ & $4,796^{*}$ \\
11 & 1,083 & 1,692 & 1,503 & $6,410^{*}$ \\
11,5 & $2,931^{*}$ & $3,664^{*}$ & $3,800^{*}$ & 1,185 \\
\hline
\end{tabular}

De acordo com as comparações feitas entre os resultados das escolas estaduais de São Paulo e Porto
Alegre, observa-se que as diferenças foram significantes em todas as faixas etárias. Os resultados foram sempre mais altos para as crianças de Porto Alegre, com exceção da faixa de 11 anos.

Na comparação entre as escolas estaduais de Porto Alegre e as municipais de São Paulo, as diferenças foram significantes em todas as comparações exceto aos 7,5, 8,0, 10,5 e 11 anos. Todas as médias foram mais altas para as crianças de Porto Alegre, exceto aos 8 anos, em que as crianças das escolas municipais de São Paulo tiveram um resultado maior, embora a diferença não tenha sido significante.

Comparando-se os resultados do Rio Grande do Sul com o total de escolas públicas de São Paulo, novamente as diferenças foram significantes, exceto aos 11 anos, com resultados maiores para as crianças gaúchas em todas as idades.

$\mathrm{Na}$ comparação com as crianças das escolas particulares de São Paulo não foram encontradas diferenças nas idades de 5,$0 ; 5,5 ; 6,0 ; 6,5 ; 9,0$ e 11,0 . Nas outras, idades, as diferenças foram significantes. Em geral, as médias das crianças de escolas particulares de São Paulo foram maiores, embora em muitos casos a diferença fosse muito pequena. Além disso, aos 6 anos as crianças gaúchas tiveram resultados maiores, ainda que a diferença não tenha sido significante.

Observando-se os dados polidos da Figura 1 pode-se constatar que as crianças das escolas particulares tiveram resultados mais altos que os de todos os outros grupos. Os resultados das crianças das escolas estaduais de Porto Alegre foram superiores aos das crianças das escolas públicas de São Paulo.

\section{DISCUSSÃO}

Os resultados desta pesquisa reforçam a necessidade de se obterem normas distintas para as diversas regiões do Brasil para o Teste das Matrizes Progressivas Coloridas de Raven, bem como para outros testes de inteligência, uma vez que no país existem grandes diferenças culturais e de nível socioeconômico. Assim mesmo, considerando crianças de escolas públicas de duas regiões diferentes, foi possível constatar diferenças significativas nos resultados obtidos no teste, o que pode estar refletindo parcialmente a grande diversidade de níveis socioeconômicos existente em São Paulo, que parece ser bem menor no Rio Grande do Sul. 
É interessante se pensar que Porto Alegre ${ }^{1}$, quando comparada a São Paulo, conforme o IBGE (2002), possui uma menor renda mensal ( $\mathrm{R} \$ 852,10$ e $\mathrm{R} \$$ 1031,10, respectivamente) e uma média de anos de estudo para sujeitos com 10 anos ou mais similar (7,5 e 7,6 , respectivamente). A maior diferença, mas que não é tanta, está na taxa de analfabetismo, que é maior em São Paulo (4,0 e 4,7 respectivamente). Portanto, provavelmente, não são variáveis desse nível que estão provocando tais diferenças.

Ainda quanto à discussão sobre as diferenças entre os resultados das pesquisas normativas brasileiras é interessante apresentar as razões fornecidas por Angelini, Alves, Custódio e Duarte (1989). Segundo esses autores, os resultados mais baixos obtidos por Angelini, Rosamilha e Almeida (1966) são atribuídos a diferenças no processo de amostragem, uma vez que neste caso a amostra foi coletada sorteando-se casas, o que fez com que na amostra fossem incluídas crianças que nunca freqüentaram a escola, crianças que abandonaram a escola e outras atrasadas em termos de escolarização. Tal fato, conseqüentemente, fez com que os resultados medianos obtidos em cada faixa etária fossem mais baixos do que os da pesquisa normativa de 1988, realizada em São Paulo.

A pesquisa de Jacquemin e Xavier (1982), como já foi mencionado, foi composta apenas de crianças que estavam na série escolar compatível com a idade, eliminando as crianças atrasadas em termos de escolaridade, o que causou um aumento nos resultados médios por idade. A pesquisa normativa de Angelini e cols. (1988) incluiu muitas crianças atrasadas na escolaridade, fazendo com que as médias fossem mais baixas do que as de Jacquemin e Xavier.

Outro tipo de explicação, que pode ser responsável pelos resultados mais altos das crianças das escolas estaduais de Porto Alegre e também pelas diferenças entre as pesquisas de 1966 e 1988, foi levantada por Flynn (1999) e por Raven, Raven e Court (1998), referente ao que é conhecido na literatura internacional como "efeito Flynn". Segundo Flynn (1999), os significativos ganhos no QI, observados ao examinar estudos normativos realizados em diferentes épocas, indicam que a presente geração tem uma grande vantagem no QI sobre a geração prévia, a qual, porém, não é acompanhada por uma vantagem correspondente nos testes de aproveitamento. Para Flynn as diferenças no QI entre gerações são claramente ambientais na origem. Dados

1 Referência às regiões metropolitanas de Porto Alegre e São Paulo relatados por este autor, obtidos a partir de 73 estudos realizados com cerca de 7.500 participantes, com idades variando de 2 a 48 anos de idade, entre 1932 e 1978, mostraram que os resultados dos americanos brancos tiveram um aumento de 14 pontos de QI, o que significa um aumento de 0,30 pontos de QI por ano. Uma discussão mais detalhada sobre esse assunto pode ser encontrada no artigo de Flynn (1999).

Raven, Raven e Court (1998) apontam também que houve um aumento na capacidade edutiva relacionada à data de nascimento, mas o mesmo não aconteceu em relação à capacidade reprodutiva. Entretanto, no caso da presente pesquisa 0 intervalo de tempo é relativamente pequeno para justificar as diferenças.

Outra explicação possível seria uma superioridade em termos do desenvolvimento da capacidade intelectual das crianças do Rio Grande do Sul sobre as de outras regiões do país, conforme foi apontado por Cunha, Carvalho, Moraes e Maraninchi (1968), ao compararem os resultados obtidos no INV Forma A, de sujeitos de 11 a 19 anos desta região com as normas publicadas no manual do teste de 1000 adolescentes que participaram da padronização deste teste no Rio de Janeiro. Resultado semelhante foi obtido por Cunha e colaboradores (citado por Campos, 1979) em um estudo realizado em 1959 com a Escala Geral do Raven, onde os resultados do Rio Grande do Sul foram comparados com os dos sujeitos de 12 a 18 anos do estudo de Angelini, Rosamilha e Almeida (1966). Deve-se lembrar ainda que esse resultado precisa ser encarado com cuidado, pois no estudo de 1966 foi usado o mesmo processo de amostragem que para Escala colorida.

Outro aspecto que pode ter contribuído para a diferença nos resultados das escolas públicas entre as duas cidades pode ser o fato de que São Paulo é uma cidade que recebeu durante muito tempo um número significativo de migrantes nordestinos, que vieram de condições ambientais e culturais muito desfavorecidas e muito pobres, o que pode ter se refletido nas normas. Fato semelhante não ocorre em Porto Alegre, onde as condições socioeconômicas e culturais são mais favoráveis e permitem um maior desenvolvimento do nível intelectual das crianças.

A questão pode, ainda, estar centrada no tipo de escola, tal como foi referido por Alves (1998), mas não devido a questões socioeconômicas, e sim, ao tipo de ensino. É provável que as escolas de Porto Alegre estejam sendo mais eficientes no treinamento da capacidade edutiva dos seus alunos do que as de São Paulo. O mesmo pode-se dizer das escolas particulares, que em São Paulo foram as que 
apresentaram resultados mais altos. Pelo que pode ser observado, ainda há muita dúvida em se explicar o porquê dessas diferenças regionais. Portanto, é importante que, nos próximos estudos de normas a serem realizados em Porto Alegre e outras cidades do Brasil, sejam levadas em consideração variáveis tais como renda e escolaridade dos pais, tipo de ensino das escolas e outras que possam interferir nos resultados de testes de inteligência.

\section{REFERÊNCIAS}

Alves, I.C.B. (s/d). Plano de coleta de dados para obtenção de normas para o Teste das Matrizes Progressivas Coloridas de Raven. (Manuscrito Não-Publicado.)

Alves, I.C.B. \& Duarte, J.L.M. (1993). Padronização Brasileira da Escala de Maturidade Mental Colúmbia. Em Burgemeister, B.B.; Burgemeister, L.H. \& Lorge, I., Escala de Maturidade Mental Colúmbia: manual para aplicação e interpretação. ( $3^{\mathrm{a}} \mathrm{ed}$.) (pp. 24-32). São Paulo: Casa do Psicólogo.

Alves, I. C. B. (1998). Variáveis significativas na avaliação da inteligência. Psicologia Escolar e Educacional, 2(2), 109-114.

Angelini, A.L.; Rosamilha, N. \& Almeida, R.M. (1966). Normas Brasileiras do Teste de Matrizes Progressivas. Ciência e Cultura, 18(2), 113-114.

Angelini, A.L., Alves, I.C.B.; Custódio, E.M.; Duarte, W.F. \& Duarte, J.L.M. (1999). Matrizes Progressivas Coloridas de Raven : Escala Especial. Manual. São Paulo: CETEPP.

Bandeira, D.R. \& Hutz, C.S. (1994). A contribuição dos testes $\mathrm{DFH}$, Bender e Raven na predição do rendimento escolar na primeira série. Psicologia: Teoria e Pesquisa, 10(1), 59-72.

Campos, F. (1979). O Teste de Raven (Escala Geral) no Brasil. En: Raven, J.C., Testes das Matrizes Progressivas - Escala Geral: Séries $A, B, C, D e E$. Rio de Janeiro: CEPA.
Cunha, J.A., Carvalho, L.C, Moraes, I.B.\& Maraninchi, S. (1968). Nível intelectual dos estudantes de escolas médias de Porto Alegre. Arquivos Brasileiros de Psicotécnica, 20(3), 39-44.

Davidoff, L.L. (1983). Introdução à Psicologia. São Paulo: McGraw-Hill do Brasil.

Figueiredo, V.L.M. (2002). Adaptação e Padronização Brasileira da Escala de Inteligência Wechsler para Crianças, Terceira Edição - WISC-III. São Paulo: Casa do Psicólogo.

Fynn, J.R. (1999). Searching for justice. The Discovery of IQ gains over time. American Psychologist, 54(1), 5-20.

IBGE. (2002). Disponível em: http://www2.ibge.gov.br/pub/ Trabalho_e_Rendimento/Pesquisa_Nacional_por_Amostra_de_Do micilios_anual/2002/. (Acessado em 18/08/2004).

Jacquemin, A. \& Xavier, M.A. (1982). O Teste das Matrizes Progressivas de Raven - Escala Especial. Estudo normativo em crianças de 5 a 11 anos. Ciência e Cultura, 34(4), 524-529.

Jacquemin, A. \& Xavier, M.A. (1984). O Teste das Matrizes Progressivas de Raven - Escala Especial. Estudo de sua fidedignidade. Ciência e Cultura, 36 (1), 77-79.

Pasquali, L.; Wechsler, S.M. \& Bensusan, E. (2002). Matrizes Progressivas do Raven Infantil: um estudo de validação para o Brasil. Avaliação Psicológica, 1(2), 95-110.

Raven, J.C.; Court, J.H \& Raven, J. (1995). Manual for Raven's Progressive Matrices and Vocabulary Scales. Section 2. Coloured Progressive Matrices. (1995 edition). Oxford: Oxford Psychologists Press.

Raven, J.C; Raven, J. \& Court, J.H. (1988). Matrizes Progressivas Coloridas de Raven. Manual. São Paulo: Casa do Psicólogo.

Raven, J.C.; Court, J.H \& Raven, J. (1998). General overview (1999 edition). Oxford: Oxford Psychologists Press.

Rosa, H.R. \& Alves, I.C.B. (2000). R-2: Teste Não Verbal de Inteligência para Crianças. Manual. São Paulo: Vetor.

Recebido em 07/04/2004 Aceito em 25/08/2004

Endereço para correspondência: Denise Ruschel Bandeira: Ramiro Barcelos, 2600 sala 120, CEP 90035-003, Porto AlegreRS.

E-mail: drbandei@terra.com.br 